\section{Histoire Épistémologie Langage}

$42-2 \mid 2020$

Genèse, origine, récapitulation. Trân Đức Thảo face aux sciences du langage

\title{
Sinologie et typologie. Deux articles de linguistique générale de Georg von der Gabelentz
}

\section{Georg von der Gabelentz}

Didier Samain (éd.)

Traducteur : Didier Samain

\section{OpenEdition}

\section{Journals}

Édition électronique

URL : https://journals.openedition.org/hel/425

DOI : $10.4000 /$ hel. 425

ISSN : 1638-1580

Éditeur

Société d'histoire et d'épistémologie des sciences du langage

Édition imprimée

Date de publication : 31 décembre 2020

Pagination : 145-173

ISBN : 979-10-91587-13-6

ISSN : 0750-8069

Référence électronique

Georg von der Gabelentz, « Sinologie et typologie. Deux articles de linguistique générale de Georg von der Gabelentz », Histoire Épistémologie Langage [En ligne], 42-2 | 2020, mis en ligne le 28 octobre 2021, consulté le 29 octobre 2021. URL : http://journals.openedition.org/hel/425 ; DOl : https://doi.org/ $10.4000 /$ hel. 425

Ce document a été généré automatiquement le 29 octobre 2021

\section{(c) $($ ) $\odot$ (8Y NO}

HEL is licensed under a Creative Commons Attribution-NonCommercial-NoDerivatives 4.0 International License 


\title{
Sinologie et typologie. Deux articles de linguistique générale de Georg von der Gabelentz
}

\author{
Georg von der Gabelentz \\ Didier Samain (éd.) \\ Traduction : Didier Samain
}

\section{Présentation des deux articles}

1 Tiré de l'oubli il y a quelques décennies par Coseriu ${ }^{1}$, qui y voyait la préfiguration de certains concepts saussuriens, Georg von der Gabelentz (1840-1893) fut d'abord un sinologue de premier plan, auteur d'une grammaire chinoise qui fit date ${ }^{2}$ et de travaux majeurs sur les langues d'Asie orientale et leur classification. Il est surtout connu aujourd'hui pour sa Sprachwissenschaft ${ }^{3}$, à laquelle on attribue plusieurs notions devenues usuelles, telles la distinction entre sujet et prédicat " psychologiques », entre synchronie et diachronie, etc., à quoi on ajoute une conception cyclique de l'évolution des langues, le recours à la statistique, l'introduction du terme typologie et, plus généralement, un effort pour définir le périmètre d'une véritable linguistique générale, sur des bases empiriques.

2 Ces quelques remarques résument sommairement le discours contemporain sur ce linguiste ${ }^{4}$. Outre le souci d'éviter les raccourcis ou les anachronismes, la difficulté pour l'historien est de saisir ce qui fait la cohérence, si elle existe, d'une œuvre balisée par deux textes majeurs et interrompue par la mort précoce de l'auteur, qui a laissé au passage des incertitudes sur la seconde édition de Sprachwissenschaft ${ }^{5}$. Par ailleurs, s'il est vrai que certains commentaires rétrospectifs n'ont pas brillé par leur acuité historique $^{6}$, force est de reconnaître qu'il n'est pas facile de cerner la situation scientifique et institutionnelle, en pleine période néogrammairienne, d'un sinologue qui héritait par ailleurs de l'anthropologie linguistique de Humboldt. Une chose est sûre, si, comme on le verra plus bas, la grammaire comparée indo-européenne servait certes de point de repère incontournable pour quiconque menait une recherche 
académique, les sciences du langage de la fin du XIX siècle n'en formaient pas moins un espace ouvert et Gabelentz, qui a publié aussi bien dans la prestigieuse Internationale Zeitschrift für Allgemeine Sprachwissenschaft que dans Indogermanische Forschungen, la revue phare des néogrammairiens, ne fut pas le savant marginalisé qu'on pourrait d'abord imaginer. De même, son effacement de l'interdiscours des linguistes ne fut pas davantage isolé, il fut strictement contemporain de celui des chercheurs de son époque, néogrammairiens en tête.

3 La première édition de Sprachwissenschaft fit du reste l'objet d'un compte rendu nuancé dans Indogermanische Forschungen par Streitberg (1864-1925), lequel estime que «si on s'enquiert des points de vue fondamentaux de l'auteur sur les principales questions de la linguistique, il est permis de répondre qu'ils ne se différencient de ceux des indoeuropéanistes sur aucun point essentiel » (Streitberg 1892: 4). Le rapport de Gabelentz à Humboldt témoigne également de la diversité du réseau linguistique de l'époque, bien loin de l'image monolithique qui en est encore parfois donnée. Georg von der Gabelentz hérite directement du programme humboldtien par son père, Hans Conon (1807-1874), humboldtien déclaré, célèbre notamment pour sa grammaire du mandchou (1832), mais simultanément salué par Leskien (1840-1916) comme le plus grand polyglotte de son temps, doublé d'un théoricien de valeur (Leskien 1875)7. En revanche, si les préoccupations anthropolinguistiques de Gabelentz et de Steinthal (1823-1899), lui aussi héritier revendiqué de Humboldt, peuvent sembler voisines, le lecteur de Sprachwissenschaft ne peut qu'être frappé par le nombre d'allusions implicites, et presque toujours critiques, aux thèses de ce dernier. On n'en citera qu'un exemple, pour sa valeur emblématique. Comme Humboldt et bien d'autres après lui et jusqu'au $\mathrm{xx}^{\mathrm{e}}$ siècle inclusivement, Steinthal défend le topos du prétendu dynamisme spécifique à la phrase indo-européenne ${ }^{8}$, tendue entre nominatif et accusatif. Une thèse rejetée par Gabelentz, qui ne manque jamais quant à lui de rétablir par contraste les droits des langues non accusatives - «si j'enfonce un clou en frappant dans le mur à coups de marteau, écrit-il (Gabelentz 1901 : 461), le clou, le marteau et le mur sont au même titre des déterminations de mon acte de frapper ». Il n'y a donc aucune contradiction logique à traiter l'objet de manière adverbiale, bien moins que lorsque nous nous glorifions de notre nominatif. En d'autres termes, le linguiste Gabelentz débarrasse ici l'anthropologie linguistique humboldtienne d'une partie de son axiologie, laquelle se trouve au contraire exacerbée chez Steinthal. Nul doute que les domaines de spécialité, tant de Hans Conon que de Georg, n'aient contribué à relativiser l'importance du modèle syntaxique indo-européen.

4 Ce ne sont là que quelques éléments de contextualisation. Une perspective plus thématique fait apparaitre encore d'autres liaisons, comme nous le verrons dans un instant. Quoi qu'il en soit, les deux textes qui suivent contribuent à leur manière à cette mise en contexte.

5 Le plus ancien est une version remaniée de la leçon inaugurale prononcée par l'auteur le 28 juin 1879 (Gabelentz 1881a), lors de sa nomination à la chaire des langues chinoise, japonaise et mandchoue qui venait d'être créée à Leipzig (dans le bastion néogrammairien !). Il s'agissait de la première chaire de langues d'Asie orientale dans une université allemande $e^{9}$. Outre les langues est-asiatiques, elle incluait un enseignement de grammaire comparée et de linguistique générale. Gabelentz y sera promu professeur ordinaire en 1882 et la quittera pour Berlin en $1889^{10}$. 
6 Cet exercice, où se mêlent par définition posture rhétorique et positions théoriques, impose à l'impétrant de préciser la finalité de cette nouvelle chaire et Gabelentz sait qu'il lui faut définir son champ et en établir la légitimité académique. Outre un panorama des études sinologiques de l'époque, l'exposé fournit une bonne illustration de quelques thématiques chères à l'auteur. Le lecteur y relève le rôle tout à la fois de modèle scientifique et de contrepoint assigné à la grammaire comparée indoeuropéenne. La Chine, se plaît-il à souligner, n'est pas l'Inde ou le Proche-Orient, ces deux figures, romantique et biblique, des origines. Son altérité embrasse la langue, la culture, et la documentation disponible. Gabelentz va jusqu'à mettre discrètement en question les principes uniformitaristes, constitutifs de la reconstruction indoeuropéenne et il en conclut que les particularités, factuelles et documentaires, des langues d'Extrême-Orient imposent au chercheur de modifier sa conception des langues. S'agissant du chinois, ceci concerne notamment la reconnaissance du rôle systémique de la syntaxe, ce qui n'allait nullement de soi dans la tradition grammaticale. Mais notre linguiste esquisse aussi des thèses bien plus provocatrices, défendant non seulement, comme son contemporain Schuchardt (1842-1927), la réalité de la mixité des langues, mais en ajoutant que, pour certains groupes de langues, les concepts habituels d'apparentement ne conviennent pas, une hypothèse que ni les néogrammairiens, ni Saussure ne se seraient risqués à avancer.

On s'étonnera peut-être a contrario du rôle assigné à la métaphore organiciste ${ }^{11}$, tout comme de la tonalité humboldtienne de certains propos, s'agissant entre autres du lien postulé entre Völkerkunde et Linguistik, alors même que le sinologue récuse le topos des langues "sans forme" et souligne parallèlement l'importance de la syntaxe. La tentation habituelle est d'y voir ce qui « date » chez Gabelentz. Certes, mais ce n'est pas si simple. C'est d'abord une question de réseau thématique. Qu'elle soit ou non abordée de façon axiologique, l'importance accordée à la syntaxe relie de fait Gabelentz à la tradition humboldtienne et, par delà Humboldt, à la grammaire générale; et elle l'éloigne parallèlement des indo-européanistes de son temps et des structuralistes de la période suivante. Par ailleurs, en fournissant un matériau qui complétait à ses yeux les données issues de la grammaire comparée indo-européenne, et/ou de la tradition grammaticale, la sinologie devait, selon Gabelentz, contribuer à l'édification d'une véritable linguistique générale telle qu'a pu la rêver Humboldt, une linguistique bâtie sur la diversité des "organismes langagiers ", c'est-à-dire, ni sur les fondements onomasiologiques des grammaires générales, ni sur le mode programmatique qu'en proposera le CLG. En ce sens, Gabelentz était bien de son époque, celle dont l'Internationale Zeitschrift für Allgemeine Sprachwissenschaft fut l'illustration la plus emblématique.

8 Les thématiques abordées dans cet article, comme ensuite dans Sprachwissenschaft volonté d'articuler anthropologie et linguistique, recours à la métaphore organiciste, question des langues mixtes et des apparentements non génétiques - sont de fait récurrentes à l'époque ${ }^{12}$. S'agissant du dernier point, on songera par exemple à l'« affinité fonctionnelle » de Pott (1802-1887) ou à la distinction entre "parenté » et " alliance » introduite par Grasserie (1839-1914) ${ }^{13}$, auquel Gabelentz se réfère dans le second texte présenté ici, qui fut le dernier article de l'auteur, mort sans avoir eu le temps d'en corriger les coquilles (Gabelentz 1894) ${ }^{14}$. Ces diverses thématiques sont cette fois centrales et associées à une ambition affichée de fonder l'allgemeine Sprachwissenschaft sur des bases aussi solides que celles de la sprachgeschichtliche 
Forschung. Vu le contexte, on peut penser que l'introduction du mot typologie fut surtout terminologique, mais il faut prendre garde aux biais rétrospectifs. En admettant que le sens de ce mot fasse consensus chez les linguistes d'aujourd'hui, celui que lui attribuait Gabelentz n'est pas d'office évident.

Inusité dans les sciences, il fait à l'époque quasiment figure de néologisme. Il est répertorié dans le Meyer, le dictionnaire encyclopédique de référence, mais sous la forme d'un simple renvoi à Typ. Dans le réseau théorique de l'époque, ce dernier mot entre par contre en résonnance avec d'autres mots tels caractère ou principe. Il repose en effet sur la thèse qu'un idiome se caractérise par "certains traits physionomiques [...] saillants " (Gabelentz, ici même), dont il est donc possible d'inférer les autres caractéristiques. Ainsi conçue, la typologie n'est donc pas tant une classification de langues que des caractères fondamentaux qui en définissent des types, ce qui est un peu différent. Elle est indissociable de la notion de système, telle qu'elle était médiatisée par la métaphore organiciste. Il en résultait que les langues sont «des structures organiques libres [dont] toutes [les] parties sont l'une à l'autre en rapport nécessaire " (ibid.). Le principe ex ungue leonem devenait alors un objectif légitime en linguistique et la référence, même distante, à Cuvier prenait tout son sens.

Quoi qu'il en soit, Gabelentz observe que des traits grammaticaux similaires apparaissent bel et bien dans des langues sans contact, ni apparentement généalogique. Or si la métaphore organiciste est valide et s'il s'agit de traits saillants, alors il est normal qu'ils apparaissent "conjoints » à d'autres traits avec un degré de fréquence statistique excluant la simple coïncidence (Zusammentreffen) et qu'il faille donc interpréter comme une liaison systémique (Zusammenhang) ${ }^{15}$. Gabelentz en fournit une illustration qu'il juge particulièrement probante: la conjonction statistique entre ergativité et distribution partagée des expansions du SN.

11 L'historien verra naturellement dans l'appel à des méthodes statistiques et normalisées le signe d'une professionnalisation d'un champ, la «typologie» ou la "linguistique générale » (les deux formules sont donc quasi synonymes pour Gabelentz), jusqu'alors plutôt exploré par des linguistes amateurs ${ }^{16}$. De ce point de vue, les deux textes qui suivent sont deux balises dans l'histoire de la disciplinarisation des savoirs sur le langage. L'épistémologue trouvera de son côté matière à réflexion en constatant que l'appel à une méthode normalisée, présentant des garanties jugées modernes de scientificité, se fondait sur une représentation datée et critiquable des langues, comme « systèmes où tout se tient $»^{17}$.

12 Formulée d'une manière ou d'une autre, cette dernière thèse est récurrente chez Gabelentz. "La langue, écrit-il dès les premières pages de la première édition de Sprachwissenschaft (Gabelentz 1891: 10), est aussi peu une collection de mots et de formes qu'un corps humain n'est une collection de membres et d'organes ${ }^{18}$. Langue et corps sont à chaque phase de leur existence des systèmes (relativement) complets, qui ne dépendent que d'eux-mêmes. Toutes leurs parties sont en interaction, et chacune de leurs expressions vitales correspond à cette interaction. Rien ne ressemble davantage à un organisme que le langage humain. » Le livre développe plus loin (Gabelentz 1891 : 457) la perspective d'une "physiognomie » langagière, basée sur l'identification de "traits saillants" (hervorstechende Merkmale) caractéristiques. La thèse d'une «implication de traits» exposée dans l'article de 1894 en découle naturellement. Ici encore, difficile de ne pas songer aux sciences de la nature, en l'occurrence à la notion de «subordination des caractères » qui fonde les grandes taxinomies de l'époque ${ }^{19}$. On 
terminera cette brève présentation sur ce parallélisme. Il permet en effet de situer plus précisément Gabelentz dans le réseau discursif de son époque. Il invite par ailleurs à rapprocher les thèses de l'auteur de certains débats contemporains.

S'agissant du réseau, Gabelentz défend, comme Schuchardt et d'autres dialectologues, la thèse de la mixité des langues, une thèse en opposition frontale aux conceptions diffusionnistes et organicistes dominantes et violemment critiquée par Meillet dans une série d'articles publiés dans les premières années $\mathrm{du} \mathrm{xx}^{\mathrm{e}}$ siècle. Il y avait là, pour quelqu'un comme Meillet, un verrou épistémologique qui, à l'inverse, se dissolvait de lui-même chez des dialectologues comme Schuchardt, ou chez des gens travaillant, comme Gabelentz, sur des aires linguistiques très différentes du monde indo-européen. Force toutefois est de relever une divergence de taille entre ces deux auteurs, car Schuchardt rejette catégoriquement toute référence, même vaguement analogique, à la démarche de Cuvier. "La langue, écrit-il en réponse à Meillet, n'est pas une masse homogène, dont un échantillon suffirait ; elle n'est pas un organisme qui autoriserait le précepte ex ungue leonem [...]. À partir d'un seul mot, nous reconnaissons la langue à laquelle il appartient, nous pensons alors pouvoir, à partir de l'origine démontrée de ce mot, tirer une conclusion sur l'origine de l'ensemble des mots qui se sont associés à lui, ou des faits linguistiques en général. [...] Pareille pars pro toto ne saurait en aucune façon se justifier comme procédé "heuristique". (Schuchardt 1976 [1928] : 195-196). Dans les pages qu'il consacre à Gabelentz, François (2017) souligne avec raison la similitude frappante entre les Konjunkturen de Gabelentz et les implicational universals. Or la question reste ouverte, justement, de savoir comment interpréter les implications proposées par Greenberg et ses disciples. Est-il par exemple possible de mettre en évidence des corrélations entre des particularités morphosyntaxiques et des traits phonologiques qui ne soient imputables, ni à une parenté historique, ni à des facteurs aréaux ? C'était là le cœur de la thèse de Gabelentz. Force est de constater qu'elle fait débat chez les typologues d'aujourd'hui et que Schuchardt n'a peut-être pas dit son dernier mot.

\section{Les études est-asiatiques et la linguistique $(1881)^{20}$}

Ce qu'on entend par "études orientales" a connu, comme on sait, une extension constante et très conséquente durant notre siècle. Du temps de nos grands-parents, les études sur le Moyen-Orient ${ }^{21}$ n'étaient encore guère plus qu'une annexe de la théologie. La langue de l'Ancien Testament en constituait pour ainsi dire le cœur et d'autres langues sémitiques, le syrien, le chaldéen, le samaritain, l'arabe, plus éventuellement l'éthiopien, s'y adjoignaient aux deuxième et troisième rangs. Et comme l'univers culturel musulman nous était devenu plus familier, il est arrivé qu'on intègre aussi le turc et le persan dans le périmètre de ces études et quelques-uns se sont aventurés jusqu'à l'arménien et au copte. Mais ils sont restés isolés. Il y avait longtemps déjà que des missionnaires chrétiens, à commencer par les infatigables jésuites, avaient entrepris de décrire les langues étrangères les unes après les autres sur le plan grammatical et lexical ; des voyageurs avaient noté ce qu'ils avaient glané en chemin dans de lointaines contrées. Ce n'est pas le matériel à récolter qui aurait fait défaut, ce qui faisait défaut était une méthode scientifique pour les récolter. On constituait bien des recueils polyglottes - on sait quelle part y a pris l'esprit universellement fécond de 
Leibniz -, si ce n'est qu'ils ressemblaient encore quelque peu aux cabinets de curiosités des anciens châteaux. Il était réservé à notre siècle de les transformer en musées.

De fait, il y fallut une puissante incitation et nous la devons à une heureuse coïncidence. La philosophie du siècle dernier avait aussi intégré le langage humain dans le périmètre de ses spéculations. Sans doute, l'entreprise était-elle fort prématurée, mais elle était assurément stimulante. Ce qui jusqu'alors avait simplement offert à la curiosité l'attrait de la bizarrerie, on apprenait à l'observer avec de tout autres yeux. Entre le Vocabulaire de Catherine II et le Mithridate d'Adelung, le progrès fut immense.

Un second progrès a suivi. En 1799, Gyármáthi s'était livré à une comparaison grammaticale d'une partie des langues du rameau fennique (ougrien), qui en établissait l'apparentement. Si son œuvre n'a pas eu une portée historique équivalente à celles qu'eurent peu après les travaux de Bopp et de Grimm, la faute n'en revient pas au travail qu'il a réalisé mais à l'objet choisi. Il fallait que l'objet fût intégralement rapatrié pour qu'il pût recevoir un juste écho. Mais le sanskrit est entré en scène. On vit avec une surprise jubilatoire une lumière totalement nouvelle se répandre sur nos langues, on reconnut dans le peuple des vieux penseurs et poètes de respectables parents de notre propre lignée. En règle générale, nos instituts supérieurs sont du genre coriace, une nouvelle discipline scientifique doit se battre pour arracher une chaire. Cette fois, cependant, les hésitations n'ont guère duré. L'élève de Bopp fut accueilli les bras ouverts. Cela ne faisait aucun doute, cette branche des études orientales n'avait rien à voir avec la théologie. Sa place se trouvait dans les locaux de la faculté de Philosophie et les autres branches, dès l'instant qu'elles ne voulaient pas servir d'abord à la critique biblique, l'y ont ensuite tout doucement suivie. Je n'évoquerai pas les grandes découvertes réalisées dans les domaines égyptien, bactrio-perse et assyrien. Bref, au final, c'est vers nous-mêmes qu'est orienté l'ensemble du champ des études moyenorientales décrit jusqu'ici : d'où nos peuples indo-européens proviennent-ils? D'où leur culture provient-elle?

Ce ne fut donc peut-être pas un hasard si nos universités allemandes ont aussitôt trouvé leur compte au sein de ce champ. Abstraction faite de ce qui vise directement la vie elle-même - la théorie politique et économique, le droit, la religion et la santé publique -, je ne vois guère ce qui devrait susciter davantage notre intérêt que la question de notre propre histoire. À vrai dire, la France, et pour le coup à la suite d'une décision très rapide, est allée tout de suite encore plus loin. Plus de 10000 volumes des livres chinois les plus importants sommeillaient sur les étagères de la Bibliothèque nationale. On pressentit qu'il y avait là des trésors qui apporteraient de nouveaux enseignements et cela a suffi. En 1814, Abel Rémusat fut nommé professeur de langues est-asiatiques au Collège de France et depuis cette époque les sinologues français ont mené le bal pendant près d'un demi-siècle, jusqu'au moment où des maîtres anglais leur ont disputé la prééminence. Ce n'est qu'en 1838 que la Prusse a suivi, avec la nomination de Schott à l'université de Berlin, et depuis ce moment l'esprit allemand a fait preuve également dans ce domaine de son talent particulier. C'est en effet aux savants berlinois que nous devons la première grammaire de cette langue vraiment basée sur une systématique scientifique. Une seconde chaire de langues et littératures d'Extrême-Orient existe depuis 1878 à l'École supérieure de Leipzig. Cette dernière m'a été confiée et ma leçon inaugurale se devait naturellement de traiter des tâches et de la justification de ce nouvel enseignement. En ce qui concerne le dernier aspect, les personnes les plus directement concernées le reconnaissent, à vrai dire, dans le fait lui- 
même. Sauf que, je le répète, l'objet semble trop éloigné de l'orientation suivie jusqu'à présent par nos études moyen-orientales et linguistiques. Chaque effort véritablement scientifique est légitime, cela ne fait aucun doute. Sauf que toutes les branches de la recherche scientifique ne sont pas susceptibles de trouver place dans le cadre des études universitaires. En l'occurrence, la décision ne relève pas uniquement du forum des spécialistes, des savants. La question n'est scientifique que pour une part, l'autre part est pratique. Comment y répondre?

On ne gagnerait guère à se contenter d'une référence à d'autres pays, pour certains plus petits, aux établissements d'enseignement supérieur de France, d'Angleterre, de Russie, des Pays-Bas et d'Italie; car, du moins en matière de science, l'Allemagne n'a pas coutume d'être celui qui doit apprendre à l'exemple de ses voisins. Cela ne nous dispense en rien de suivre attentivement les réalisations étrangères. Ignorer cet impératif risquerait de nous fermer certains de ces chemins prometteurs dont d'autres font leur profit. Et si maintenant je regarde ce que les savants d'autres nations et ce que certains de nos compatriotes ont ramené des mines inépuisables des littératures estasiatiques, il me faut former le souhait de voir s'activer une solide dose d'effort allemand et d'esprit allemand. Les peuples d'Asie orientale entrent aujourd'hui en contact avec le monde culturel euro-américain, ils le font d'une autre façon qu'à l'époque des grandes migrations, de façon pacifique, mais non moins vigoureuse. Une compétition de plus en plus vive a commencé. Le peuple japonais, talentueux et énergique, s'est jeté au cœur du génie européen d'un bond qui eût été un saut de la mort pour toute autre nation. Le Chinois, jusqu'à présent moins accessible à nos idées qu'à nos mines d'argent, exige en contrepartie de l'accueil de nos marchands, nos diplomates et nos missionnaires, l'entrée libre de ses bataillons d'ouvriers dans les ateliers de notre activité manufacturière. Plus prometteurs que tous les autres Asiatiques, ces gens de l'Orient extrême nous sont bien plus proches du point de vue politique et économique que les Hindous, intelligents mais passifs, ou ceux qui se réclament de l'Islam, qu'ils soient de sang aryen, tatar ou sémitique.

Il s'agit d'aller chez eux et de s'y retrouver, de comprendre leur façon de penser et de vivre. L'histoire des derniers siècles a montré à quel point tout malentendu peut ici conduire aux erreurs les plus graves. C'est ici qu'incombe aux savants, aux spécialistes des peuples et des langues, un rôle de pionnier qu'il m'est difficile d'imaginer plus gratifiant. Ni plus gratifiant ni plus attrayant. Je pense à moi et à ma spécialité et avant tout à l'étude de la civilisation chinoise, telle qu'elle se reflète dans l'une des plus intéressantes littératures au monde. Cette littérature, fondée il y a plus de 4000 ans, et donc la plus ancienne des littératures vivantes, plus diverse qu'aucune autre en Orient, peut-être plus abondante même que la plupart des littératures européennes, nous est à peine connue par quelques-unes de ses productions, et encore, combien ces dernières sont-elles mal connues!

Force hélas m'est de reconnaître une chose : on conclut trop volontiers de l'importance de la demande à la qualité de la marchandise. Tout le monde sait à quel point ce raisonnement est trompeur en matière littéraire. Ici le meilleur est pour les meilleurs, donc pas toujours pour la majorité, et même le meilleur doit conquérir sa place, dès lors qu'il est quelqu'un d'isolé, d'inattendu. Il ne faut pas s'illusionner : la sinologie va mal en Allemagne. Nous cultivons d'autres fruits dans le champ de nos études orientales, et avec quels brillants résultats! Nos études indiennes, iraniennes, sémitiques et égyptologiques délivrent au monde savant de magnifiques réalisations 
qui intègrent, en quelque sorte, son patrimoine. Les pharaons et leurs vassaux, les musulmans et les brahmanes nous font presque l'effet de vieux compagnons et de cousins, avec lesquels on ne saurait trop rapidement se mettre à tu et toi et dont nous écoutons les histoires comme s'il s'agissait des contes de notre propre enfance. Les échos qui nous parviennent depuis le monde culturel chinois n'évoquent, à vrai dire, aucun son familier. Les choses se sont développées de part et d'autre de façon tellement indépendante que les deux parties ne peuvent guère avoir en partage que ce qui fait leur humanité commune. Et pourtant, c'est énorme! Qu'on brise la coquille exotique, que l'on pénètre dans la signification de cette riche poésie, dans ses passions, dans sa méditation, son aspiration, son humour, on oubliera alors assez vite que près des deux tiers des individus de notre hémisphère ont migré vers l'est. Assez vite notre imagination apprend à tracer des traits sympathiques sur les visages chinois lisses, jaunes et aux yeux bridés, et ce qui ressemblait de loin à une poupée de bois, s'anime et révèle alors son humanité chaude et sensible.

Ou bien, tentons de nous immerger dans les secrets de la philosophie chinoise, dans sa profonde mystique, dans ses courants optimistes et pessimistes, réalistes et idéalistes, dans les combats de ses systèmes, dans l'histoire de son développement permanent. Si nous inférons la valeur de cette gigantesque bibliothèque que nous annoncent les catalogues indigènes à partir du peu qui nous est actuellement accessible, alors nous aurons la surprise de découvrir, à la place de ce tableau d'uniformité intellectuelle qu'on nous a brossé d'avance, le théâtre d'un combat intellectuel violent et nous remarquerons sur le front jaune du Chinois les profondes rides du penseur. Le philosophe exceptionnellement laconique dont l'esprit domine depuis deux mille ans le tiers de l'humanité était un Chinois et il faudrait connaître l'enseignement de Confucius avant de formuler un jugement sur l'Empire du Milieu et ses habitants.

Si nous évaluons l'importance d'une personnalité historique selon la force, l'étendue spatiale et temporelle de son influence immédiate et ultérieure, Confucius compte parmi les grands hommes de toutes les époques, au nombre des plus grands. Mais j'estime qu'il est encore à bien des égards gravement méconnu. On veut encore et toujours le classer parmi les fondateurs de religion. Rien d'étonnant qu'on ne lui rende pas raison de la sorte. Il n'était certainement pas irréligieux, comme il pourrait éventuellement en donner l'impression à l'observateur superficiel. Il croyait au caractère sacré des devoirs humains et à la justice de la providence divine, qui punit et récompense selon le mérite. Mais il manquait à son esprit la disposition et la tendance à la mystique. Il ne connaissait, si je puis user de cette expression moderne, que « Dieu dans l'histoire». Son esprit était essentiellement pratique et donc historique. "Je ne produis rien de nouveau ", disait-il de lui-même, " je transmets. Je crois aux anciens et je les aime. » Mais il ne transmettait que ce qui avait fait ses preuves. Il connaissait son peuple comme probablement nul autre et reconnaissait ce qui lui serait à tout jamais utile. C'est là que je vois sa grandeur: c'est la grandeur de l'homme d'État et du philosophe pratique.

II est notoire qu'aucun État sur terre n'a mieux veillé que l'Empire chinois à l'exhaustivité et à la fiabilité de ses annales. Depuis la plus haute antiquité, les gouvernements ont payé des hommes d'État lettrés dont la tâche était, et reste, de consigner de façon indépendante chaque événement mémorable. Les documents enregistrés par ces derniers étaient conservés dans une archive secrète inaccessible même au prince et à son cabinet, et n'étaient mis au jour et utilisés qu'après la chute de 
la dynastie. C'est ainsi que s'est constituée cette œuvre de centaines et de centaines de volumes qui forme les annales royales chinoises et témoigne d'une histoire quadrimillénaire. Y sont associés, en les complétant, des travaux spécifiques sur l'histoire et la géographie des provinces et des districts, dont un seul remplit 160 solides cahiers, de même que d'énormes travaux biographiques et d'histoire littéraire. Et quoi qu'on puisse encore nous dire de l'immobilité et de la stagnation du monde chinois, l'Empire et son peuple ont une histoire, au cours de laquelle des idées se sont développées, des formes d'État, des usages et des statuts sociaux se sont transformés, de nouvelles inventions et organisations se sont imposées, plus lentement que chez nous peut-être, mais avec à peine moins de force. À tel endroit on nous parle du renforcement progressif des États féodaux indépendants, d'une situation séculaire de guerre et de conflit, amenant dévastation et décadence des mœurs, comme le fut notre guerre de Trente Ans. Puis du sauvetage de la société par l'œuvre réformatrice de Confucius et de son école. Puis à nouveau du système radical de gouvernement mis en place par l'empereur Qin Shi Huang, ou de cet État socialiste éphémère du temps de la dynastie Song, et de tant et tant d'autres expérimentations de gouvernement, pour lesquelles il ne faut pas toujours chercher bien loin des analogies dans notre propre histoire. On reproche aux Chinois le manque de vaillance guerrière. Non à tort, semblet-il. En effet leurs performances militaires face à des armes européennes ont été jusqu'à présent assez piètres. Sauf que je pourrais nommer à partir des annales d'une seule dynastie deux généraux qui ont accompli avec leurs hommes la même chose que ce qui a valu une gloire immortelle à Léonidas et à ses Spartiates. Et ce qui suit est en quelque sorte le modèle pour toute une série d'épisodes de l'histoire chinoise. L'empereur annonce une intention en conférence de cour, un ministre le contredit; l'empereur n'accorde aucune attention à ses objections et - ainsi s'expriment les historiens - «le ministre meurt ». Ai-je besoin d'ajouter quels éclaircissements nous devons espérer de ce côté-là pour la géographie et l'histoire de l'Asie orientale et pour l'origine des grandes migrations? Nous connaissons la force expansionniste obstinée du peuple chinois et il nous est permis de deviner comment sa constante progression vers le nord et vers l'ouest a pu se transformer chez ses voisins nomades en ruée dévastatrice.

L'imprimerie est généralisée en Chine depuis le dixième siècle de notre ère. Le peuple est devenu depuis cette époque l'un des plus enclins à la lecture et l'écriture. L'aspiration à la culture est ici plus répandue que dans certains pays de notre continent. L'érudition seule donne droit au rang et à la puissance et j'apprends que ces gens les plus pauvres du peuple qui tentent leur chance en Californie ont emmené leurs maîtres d'école et leurs libraires avec eux dans leur nouvelle patrie. Toutes les classes, mais aussi toutes les provinces et les ethnies de la nation apportent leur part au travail littéraire, et une saine force décentralisatrice venue d'en bas fait contre-poids à l'influence uniformisante venue d'en haut. Des empereurs éclairés font imprimer des éditions de luxe des œuvres majeures. Le gouvernement anglais s'est récemment procuré l'une d'entre elles en 10000 volumes. De riches particuliers se font une gloire de financer de leurs propres deniers de nouvelles éditions de leurs livres préférés et de les diffuser pour une bouchée de pain. Je pose maintenant la question : est-ce que tout cela suggère l'indolence, l'enlisement intellectuel?

Je ne parlerai pas à mes lecteurs des réalisations des Chinois pour la connaissance de leur langue, de leurs dictionnaires en deux cents volumes, de leurs gigantesques travaux de critique philologique. À quoi bon au demeurant les chiffres, qui font silence sur ce qu'il y a de meilleur? Je préfère leur ouvrir une fenêtre sur ce domaine heureux 
de la nouvelle littérature qui est foisonnante. Elle est le miroir le plus fidèle de la vie actuelle du peuple, comme ce dernier, elle est multicolore, multiforme et aussi, à l'occasion, immonde, ici pédantesque et raffinée à l'excès, là se livrant à tous les excès dans un laisser-aller génial. Un humour incroyable, souvent brillant, suivi à nouveau d'une poésie émue, authentique, de fantaisies féériques, puis derechef le réalisme intégral empreint de subtile vérité psychologique, tout cela le chinois le possède dans les plus bénignes de ses productions intellectuelles.

Il n'est actuellement pas possible d'évaluer quel apport l'étude du bouddhisme peut espérer de ses sources chinoises et japonaises. Les récits de voyages de moines hardis, auxquels Stanislas Julien nous a donné accès, ont recueilli l'intérêt qu'ils méritaient auprès des cercles d'indianistes. Une traduction du Tripițaka, en largement deux mille cahiers, récemment parvenue en Angleterre, attend pour le moment d'être exploitée.

Notre sinologie est redevable à plus d'un titre à la domination étrangère des Mandchous en Chine. Nullement à cause de la littérature mandchoue. Cette dernière n'est pas très riche et n'est un produit intellectuel autochtone que pour une infime partie. Mais ses traductions de tant d'œuvres chinoises les plus importantes dans une langue facile à apprendre et pourvue d'une écriture alphabétique commode doivent être considérées comme quasi authentiques et, là où elles sont disponibles, elles sont pour nous encore aujourd'hui une aide inestimable. Aucun sinologue européen ne peut se permettre d'ignorer le mandchou.

En ce qui concerne la production intellectuelle de la littérature japonaise, nous pouvons davantage la juger par son contenu et sa valeur. Il est une chose que l'on reconnaît déjà aujourd'hui : quels que soit l'enthousiasme et le succès avec lesquels cet étonnant peuple insulaire a ingéré la culture de l'Empire du Milieu, il a emprunté, sur bien des points, des chemins qui lui sont tout à fait spécifiques. La tournure d'esprit des deux nations était trop différente, celle des Japonais était déjà trop développée et consolidée lorsque l'univers chinois l'a pénétrée. Les Japonais l'ont absorbé en eux au lieu de se fondre en lui. Une remarquable mythologie, débouchant sur l'histoire proprement dite du pays, puis cette dernière elle-même, constituent le contenu des œuvres remarquables les plus anciennes. Bientôt, on esquissa aussi ces courtes et gracieuses histoires lyriques dans lesquelles le peuple chevaleresque et passionné aime exprimer ses états d'âme. La vie même invitait suffisamment au romantisme, il ne s'agissait, comme dit notre poète, que de s'en saisir vivement pour produire la matière des romans et des épopées. C'est ainsi que sont nées ces innombrables œuvres à demi historiques qui nous emplissent tour à tour d'enthousiasme et d'effroi frémissant, ces «Monogatari » saisissants et les courts récits populaires dont quelques-uns sont depuis peu portés à la connaissance du monde lettré européen. Des œuvres de pure fiction s'y sont adjointes, et parmi elles, à en juger par les échantillons qu'on m'a présentés, de véritables œuvres d'art. Une masse d'ouvrages descriptifs ou didactiques, généralement pourvus d'illustrations sommaires mais claires, nous conduisent dans les paysages du magnifique archipel, dans sa faune et sa flore, ou dans les activités commerciales ou agricoles de ses habitants.

Les travaux des sages chinois et les écrits des bouddhistes ont reçu un accueil enthousiaste au Japon. Pour les jeunes gens cultivés de ce pays, les classiques de l'Empire du Milieu furent depuis cette époque exactement ce que sont les classiques grecs et latins pour nos lycéens. Ils étaient même encore davantage et le siècle de notre humanisme nous revient en mémoire, lorsque nous apprenons que le Japonais de jadis 
passait tout au long de sa vie pour d'autant plus cultivé qu'il était plus familiarisé avec cette littérature étrangère, qu'il était plus à l'aise dans le style chinois. Une telle dépendance ne pouvait que devenir franchement funeste pour la science autochtone. Pour autant que je sache, on n'a encore jamais entendu parler d'un philosophe japonais indépendant et il n'y aurait rien d'étonnant à ce qu'il n'y en ait jamais eu un. La manière dont les Japonais rendent comestibles pour leurs compatriotes les fruits intellectuels étrangers n'en est que plus séduisante. Parmi ces prédicateurs populaires qui parcourent le pays depuis nombre d'années et exposent devant des ouvriers, des femmes et des enfants les plus belles maximes tirées des trois enseignements officiels, on trouve de véritables maîtres en inventio et dispositio rhétoriques.

La critique philologique des anciens écrivains est à peine moins florissante au Japon que dans l'Empire du Milieu et beaucoup a été fait pour l'étude de la langue du pays. Cette dernière s'est développée et s'est transformée plus rapidement peut-être qu'aucune des autres langues de culture au monde. Les enregistrements écrits de leurs plus anciens monuments sont de telle sorte qu'ils ne donnent accès à leur compréhension scientifique que par le biais des reconstructions les plus sagaces, et ce que les Japonais ont réalisé dans cette direction mérite tous les éloges malgré quelques monstruosités. Je ne m'étendrai pas ici sur leurs nombreux et, pour une part également, très volumineux dictionnaires, dont le chercheur européen apprend tôt lui aussi à reconnaître les mérites. On connaît moins en revanche l'ampleur et l'importance de leurs travaux grammaticaux. Peut-être les livres indiens introduits par les bouddhistes leur ont-ils fourni une première impulsion en la matière, mais l'influence de ces derniers ne peut guère avoir excédé ce qu'on peut légitimement leur attribuer. Si nous considérons la violence avec laquelle il arrive encore qu'on torde chez nous les membres des langues les plus hétérogènes pour les faire entrer de force dans l'uniforme universellement prisé des langues latines, nous rendrons justice aux linguistes japonais et leur pardonnerons quelque bizarrerie eu égard au génie qui caractérise leur intelligence grammaticale et à leur ardeur à rassembler des données. Aujourd'hui, une école européenne dispute également la palme en la matière à l'école autochtone, et il y a peu, le même courrier m'a apporté du Japon deux manuels élémentaires de langue représentatifs des deux parties. Comme il est passionnant d'assister à une bataille de ce genre !

31 Un champ immense est ainsi ouvert à perte de vue aux études est-asiatiques. Leur tâche est d'abord philologique dans l'acception la plus large du mot. Mais il s'y associe une seconde tâche non moins importante : j'entends la tâche linguistique ${ }^{22}$, l'élargissement et l'approfondissement de notre connaissance de la nature du langage humain.

Dans la mesure surtout où une responsabilité m'incomberait à moi-même et à mon objet, et dès lors que je me fais l'avocat d'une cause, il se pourrait, il pourrait sembler, que je joue les procureurs. Rien en fait ne m'est plus éloigné qu'une telle intention. Il me faut partir de lieux communs pour éviter cette fâcheuse apparence.

33 La linguistique [Linguistik] comprend la connaissance scientifique des langues humaines. Pour que cette connaissance soit complète, elle doit investir son objet dans toutes les directions.

34 Chaque langue est en premier lieu un existant et elle est, à chaque stade, à chaque instant de son existence, un être complet en soi. On a parlé d'organisme de la langue pour rejeter ensuite cette expression, car elle désigne comme un être doué d'une vie propre, ce qui n'est qu'une fonction. Mais ce qui ne convient pas comme définition peut 
néanmoins, pour cette raison même, faire office de comparaison, et je n'imagine rien en fait qui puisse mieux figurer le développement et la nature du langage que, justement, l'organisme. Ici comme là tous les membres sont nécessaires l'un à l'autre et à l'ensemble et chaque fois qu'un locuteur s'exprime, il exprime simultanément toute la langue disponible en lui. Qui veut étudier l'histoire externe des langues doit comparer les manifestations de la période langagière actuelle avec les stades antérieurs analogues. Mais il faut mettre en relation entre elles toutes les manifestations d'une seule et même phase dès lors qu'on veut comprendre les forces agissantes du développement langagier. Il est hautement significatif pour notre science que ce soit précisément l'indo-européanisme qui mette actuellement l'accent sur ce qu'on appelle la fausse analogie. Un pont a été jeté sur le fossé, qui semblait encore béant il y a peu d'années, entre ce dernier et les autres branches de la linguistique générale [allgemeine Sprachwissenschaft], depuis qu'il s'est mis de son côté à étudier les forces psychologiques de l'édification du langage avec ce matériel empirique bien conservé dont il dispose en abondance.

Que ce fossé soit apparu, que son élargissement mettait en danger l'objet commun, qui voudra le nier? Les études en généalogie des langues depuis Bopp, stimulées par la multitude des forces de premier plan, devançaient largement tous les autres efforts dans le domaine linguistique. Il nous faut apprendre auprès des indo-européanistes, nous devons nous approprier leur méthode, leur critique, si nous voulons réaliser pour la connaissance d'autres familles de langues quelque chose d'équivalent à ce qu'ils ont fait pour la leur. C'est une chose que nous n'avons pas le droit d'oublier et, dans cette mesure, mais dans cette mesure seulement, nous devons reconnaitre leur supériorité. Si les nôtres sont moins nombreux, s'il nous faut travailler avec des moyens plus modestes, notre travail s'en trouve d'autant plus difficile, notre champ d'autant plus vaste, mais notre moisson s'en trouve aussi d'autant plus variée.

Mais pour qui veut entreprendre des comparaisons généalogiques sur des aires linguistiques éloignées, il n'est pas toujours facile de se confronter avec la grammaire comparée indo-européenne. Celle-ci affiche une sûreté de méthode sans cesse croissante, qui apparaîtra à celui-là aussi comme un idéal à atteindre. Mais s'il veut s'y astreindre prématurément, celle-ci peut devenir une chaîne qui va l'entraver, voire se muer en piège. Les lois qui déterminent les évolutions phonétiques et morphologiques sont elles-mêmes déterminées à leur tour par un nombre donné de facteurs historiques, ethnologiques, psychologiques et peut-être physiologiques. Les langues de notre rameau forment sans doute, tout bien compté, environ le vingtième de toutes les langues de la terre. Qui pourrait dénommer et dénombrer toutes les forces qui y ont contribué par leurs divisions et leurs migrations? Qui ira prétendre que les autres familles de langues n'auraient pas en outre subi l'action de forces totalement différentes, stimulante à tel endroit, inhibante à tel autre ? Et s'agissant des phonèmes, la capacité articulatoire a-t-elle nécessairement été partout et en tout temps aussi forte que chez nous? J'ai quand même l'impression que le Polynésien, avec ses dix à douze consonnes, est plus pauvre en la matière que nous, et que plus d'un peuple montagnard du Caucase, avec la multitude de ses phonèmes et de ses combinaisons phonétiques est peut-être encore plus riche. Alors que nous voyons les formes de la conjugaison disparaître chez nous et chez de nombreux de nos proches, nous pouvons observer ailleurs, par exemple chez les Kalmouks, comment naissent les désinences personnelles du verbe. De fait, si nos langues présentaient toutes les possibilités de développement linguistique, elles ne ressembleraient pas à un mobilier bien ordonné, destiné et adapté 
à la satisfaction des besoins de son utilisateur, mais plutôt à un gigantesque musée dont la majorité des objets donnent plutôt envie de les regarder que de les manipuler.

Ce fait précisément, la diversité des langues, nous est occasionnellement contesté, y compris par ceux qui ont laissé vagabonder leur regard au-delà des frontières locales. Il y a seulement quelques années, lisant l'ouvrage d'un célèbre érudit étranger, j'y ai encore vu évoquer une grammaire commune à toutes les langues agglutinantes et une grammaire analogue pour toutes les langues isolantes. Je connais en pratique deux façons d'assimiler ces grammaires communes : c'est, soit de n'apprendre à partir de chacune de ces classes qu'une seule langue, soit, ce qui est encore plus simple, de n'en apprendre aucune! La vérité est que dans les deux classes, à côté de langues très pauvres et grossières, il s'en trouve d'autres d'une richesse et d'une finesse insoupçonnées et que ce qu'on appelle la classe agglutinante n'est guère plus qu'un cache-désordre ${ }^{23}$, un grand fourre-tout scientifique, dans lequel on stocke provisoirement de manière uniforme des formes linguistiques fondamentalement différentes, comme le sont par exemple les différentes plantes des champs dans le foin.

Ce genre d'imprudences se corrige de soi-même. Il est plus difficile de répondre à une autre objection. À quoi bon, demande-t-on, observer l'ensemble de ce tableau bigarré ? Si je connais un certain nombre de langues issues de toutes les parties du monde, qu'est-ce que je possède de plus qu'une sorte de cabinet de curiosités? Quel est le lien interne? Où se trouve la science? On [nous] concèdera du moins une chose. Ce que raconte le Zoulou, ou le Hottentot, le Huron ou le Quechua, le Malais ou le nègre australien, tout cela c'est du langage humain, ou plus précisément, tout cela ce sont des expressions différentes de la capacité langagière humaine. Et je demande maintenant, quant à moi : cette capacité langagière est-elle ou non l'objet de la linguistique ? Et si c'est le cas, ne doit-elle pas être définie par la linguistique? Mais comment la définir tant qu'on ignore toute l'étendue de ses expressions, tant qu'on ne connait pas toutes les possibilités de développement du langage? Les chercheurs dans les sciences de la nature nous enseignent quotidiennement que les organismes les plus élémentaires ne sont pas moins importants pour la science que les organismes plus complexes. Nos étymologistes cherchent à découvrir dans la forme linguistique plus développée la forme plus primitive censée en être une étape dépassée, et celui qui veut appliquer la théorie de Darwin à la science du langage doit admettre en toute logique qu'il y a des millénaires, nos langues aussi n'étaient guère plus riches ou raffinées que celles des peuplades les plus grossières que nous connaissons. Il faudrait bien de l'imagination pour se représenter un état originel de ce type en l'absence de modèles vivants et nos anatomistes du langage peuvent espérer quelque inspiration de ces exemplaires les plus pauvres et les plus faibles du monde langagier.

39 Et une langue doit-elle donc être obligatoirement morte pour mériter notre intérêt? Ou faut-il que sa préhistoire soit accessible pour que son étude en vaille la peine ? Vu l'âge qu'on estime aujourd'hui être celui de l'espèce humaine, et ce qu'on sait de la variabilité du rythme évolutif des langues, il est permis de douter que ce qui sonnait il y a quatre mille ans dans la bouche de nos ancêtres ait davantage ressemblé aux formes les plus anciennes du langage humain que telle ou telle forme des langues actuellement existantes, ou par exemple, que le babil de nos enfants.

C'est là un point de vue accessoire, car on n'étudie pas une forme langagière donnée pour mieux comprendre cette forme ou une autre, mais pour y gagner une vision plus riche, plus complète, plus juste de la diversité des organismes langagiers. Il va de soi 
que, pour atteindre ce but, il ne suffit pas de tremper le bout des lèvres dans telle ou telle grammaire. Ce n'est que lorsqu'une langue est devenue une partie de notre moi que nous pouvons juger d'elle, de ses avantages et de ses faiblesses. C'est à vrai dire le contraire que nous pouvons lire dans un livre très apprécié et diffusé : un satisfecit que s'attribue la légèreté et qui cherche ses émules !

Que l'ethnologie et la linguistique exigent une investigation de toutes les familles de langues aussi minutieuse que celle actuellement menée pour le rameau indo-européen, cela ne devrait pas nécessiter démonstration. Des avancées notables ont déjà été accomplies dans cette direction, mais il en reste bien davantage à faire. Le fait qu'il faille le plus souvent nous passer du secours des langues anciennes doit nous inciter à être doublement prudents, à progresser avec la plus grande précaution du plus proche au lointain. À condition de suivre une méthode scrupuleuse, le succès sera forcément au rendez-vous. Mais le gain sera double. Nous y gagnerons en premier lieu une connaissance plus complète et plus exacte de la manière dont les rameaux linguistiques se délimitent à l'externe et se divisent à l'interne. Mais aussi ensuite - ou alors je me fourvoie complètement -, un enrichissement de la philosophie du langage elle-même. Le rameau indo-européen semble en particulier ne présenter qu'une fraction des possibilités d'apparentement et de direction de développement. Le développement qui nous en est connu a été qualifié - j'ignore dans quelle mesure c'est légitime - de "descendant", et on y conteste encore aujourd'hui la présence de véritables langues mixtes. Il est certain que de nombreuses autres familles de langues témoignent d'une conscience plus vive de la valeur des éléments constituants ${ }^{24}$ que la nôtre. Il se fait justement que je travaille actuellement, dans le prolongement des recherches mélanésiennes qu'avait initiées durablement mon père, sur un groupe de langues pour lesquelles il n'y a strictement rien à tirer des concepts de parenté qui nous sont familiers. S'il nous faut, ici ou quelque part ailleurs, reconnaitre d'authentiques hybrides, la conclusion s'en suit d'elle-même que deux langues peuvent être apparentées à une troisième sans l'être entre elles : des cousinages de langues, s'il est permis d'utiliser cette expression. Ces pauvres langues de contact, qui naissent encore aujourd'hui quasi sous nos yeux, le créole, le pidgin anglais et tant d'autres, suscitent un grand intérêt lorsqu'on tente de parvenir à comprendre pourquoi justement tels éléments y sont empruntés à une langue et tels autres à l'autre. Il s'agit ici de découvrir des règles qui ne font pas moins partie de la linguistique que, par exemple, celles de la mutation consonantique.

L'époque des grammaires [Sprachlehre] générales ou philosophiques au sens de Becker est révolue. Mais ce que nos ancêtres voulaient tirer spéculativement de leur propre réflexion, nous devons avec nos successeurs chercher à le gagner par un travail inductif. Le problème de la grammaire générale [allgemeine Grammatik] demeure. Des travaux comme nous en avons sur le duel, les méthodes de numération, le pronom relatif, le passif, etc., sont des contributions à sa solution. Juste quelques exemples pour montrer à quel point il y a peu à gagner, en l'occurrence, par une voie aprioristique. La langue des insulaires d'Errub et de Murray ne connaît que les nombres fondamentaux un et deux et ne peut compter que jusqu'à cinq, qui se dit deux-deux-un. Les Chiquitos d'Amérique du Sud ne connaissent pas le moindre nombre, pas même le deux. Pour exprimer la numération, d'autres langues mélanésiennes possèdent un triel en sus du singulier, du duel et du pluriel. À l'aide de leur triple passif, les langues des Philippines et de quelques autres peuples malais ont la possibilité d'élever au rang de sujet de la phrase non seulement l'objet, mais aussi le lieu et l'instrument du procès. Les habitants 
noirs d'Anatom, une île des Nouvelles-Hébrides, ne conjuguent pas le verbe, mais le pronom. Et quelle imagination pourrait bien concevoir la possibilité de ces systèmes de conjugaison monstrueux que nous présentent les langues polysynthétiques incorporantes des Indiens d'Amérique, ou le basque sur notre continent? Pour ne fournir qu'un seul exemple, les Chiriquís, ce peuple indien intéressant, qui s'est montré si enclin et capable d'accueillir la culture européenne, expriment l'idée « je pars afin de relier de nouveau ici et là » par un seul mot gälostisanidolega. Pour appréhender le langage humain dans toute la richesse de ses configurations possibles, il nous faut une vue d'ensemble sur les moyens phonétiques, morphologiques et syntaxiques de toutes les langues et sur le comportement de chacune d'entre elles à l'égard des contraintes logiques et psychologiques.

Il va de soi que ce dernier objectif ne peut être complètement atteint que lorsque toutes les langues, ou du moins toutes celles présentant un intérêt typologique [in typischer Hinsicht] majeur, auront fait l'objet d'une étude grammaticale exhaustive. Et la production de grammaires particulières représente une tâche dont on ne dira jamais assez l'importance. L'histoire de notre propre philologie peut en témoigner - il suffit de penser aux siècles de travaux préparatoires qu'il a fallu pour que la grammaire latine acquière sa constitution actuelle. Chaque forme de structure langagière réclame une forme d'exposition particulière, qui lui soit absolument spécifique. La trouver ne présuppose pas seulement d'avoir vraiment intériorisé l'idiome, mais aussi, voire principalement, une intelligence philosophique du langage sûre et claire. Lorsque l'une ou l'autre de ces qualités fait défaut à l'auteur, qu'on parcoure rapidement son travail et qu'on folâtre d'autant plus librement dans la langue vivante, dans la lecture de textes et, quand c'est possible, dans l'échange oral.

Toute acquisition d'une langue dont l'esprit nous est étranger est simultanément un acte de libération d'autant de préjugés dans lesquels les idiomes précédemment étudiés nous avaient empêtrés. On découvre des facteurs langagiers qui nous paraissent inutiles, et il en est d'autres que nous ne retrouvons pas et que nous pensions indispensables. Pourquoi cela? Parce que nous sommes depuis toujours accoutumés à observer les parties avant la totalité, les mots et leurs formes avant la phrase. Aussi longtemps qu'on ne connaît une langue que de cette façon, telle qu'elle apparaît démembrée sur la table de dissection, on n'a pas la moindre idée de ce dont elle est capable. On assène alors des jugements comme nous pouvons souvent en lire, des jugements aucunement plus sensés que le suivant: le poisson est dépourvu de poumons, donc il ne peut pas respirer! Tant que nous bornons nos études aux groupes de langues qui nous sont intellectuellement et physiquement apparentés, nous courons le risque de sous-estimer l'importance du facteur syntaxique. Le simple premier pas sérieux au-delà de cette barrière nous amènera déjà à reconnaitre à quel point nous avions jusqu'alors possédé et inconsciemment utilisé ce qui nous refuse soudain le service. Nos grammaires sanskritistes ne pourraient se permettre de s'arrêter là où la syntaxe devrait commencer, si les lois fondamentales de la structure phrastique n'étaient pas les mêmes en vieil indien que dans les autres langues appartenant à cette famille.

Je pense qu'il est temps pour nous aussi, Allemands, de nous occuper de ces domaines moins exploités de la linguistique [Sprachwissenschaft]. Ces derniers aussi demandent qu'on les exploite, personne ne le contestera, et je pense qu'ils sont en droit d'attendre 
cette exploitation d'une école supérieure de premier plan, dont la mission et la gloire sont d'être une Universitas Litterarum dans la pleine acception du terme.

Peut-être ce plaidoyer pro domo n'était-il même pas nécessaire. Il reste qu'élargie comme je le souhaite, la science du langage n'a pas de discipline plus voisine que cellelà même qui a pour objet les aires linguistiques les plus éloignées. On peut certes apprendre le chinois d'un point de vue purement pratique et empirique, comme tout autre langue. Mais on ne peut l'étudier scientifiquement, l'appréhender, sans une réflexion philosophique rigoureuse sur le langage. On a qualifié cette langue de «sans forme", parce que c'est une langue isolante, dépourvue de moyens de flexion ou d'agglutination. Et on l'a de nouveau qualifiée de langue à forme parce qu'elle est en mesure d'exprimer les relations grammaticales de manière pure et subtile comme presque aucune autre. Les deux propositions sont vraies, si incompatibles qu'elles puissent paraître. Le chinois ne possède que deux facteurs grammaticaux : un ordre des mots régis par des lois stables et claires et des mots auxiliaires assurant un rôle d'explicitation. Cela nous conduirait trop loin si je voulais expliquer, fût-ce allusivement, avec quel art et cependant quel naturel ces deux facteurs interagissent. Bref, la langue, telle qu'elle est, s'est maintenue comme le support de l'une des littératures les plus considérables, capable de n'importe quelle abstraction logique, susceptible d'une riche structure périodique, puis à nouveau de concision violente, de force et de profondeur rhétoriques, telles qu'il pourrait difficilement s'en trouver ainsi réunies dans une autre langue. La grammaire exige fort peu de la mémoire de l'apprenant, elle exige énormément de sa pensée logique. La récitation des paradigmes et l'énumération des irrégularités lui sont épargnées. Il lui faut pour cela accommoder son esprit à un mode de pensée qui lui est tout à fait nouveau. Il lui faut non seulement le comprendre dans toute sa cohérence, mais véritablement en faire l'expérience, et je ne sache pas d'endroit où l'importance de la syntaxe pourrait lui apparaitre plus clairement qu'ici, où ce ne sont pas seulement les relations des mots entre eux mais aussi leur fonction en tant que parties du discours, en tant que substantifs, adjectifs, verbes, etc., qui résultent uniquement du nexus phrastique. Sans doute l'incitation estt-elle du genre exclusif, mais elle est du genre puissant; celui qui l'a éprouvée avec un esprit scientifiquement réceptif, celui-là éprouvera d'office l'envie d'autres incitations analogues. Et aucune envie n'est mieux fondée; avec chaque nouvelle langue que nous étudions s'ouvre en effet en nous un nouvel univers mental.

\section{Typologie des langues, une nouvelle tâche pour la linguistique (1894)}

Si la linguistique générale doit répondre à la question "d'où vient la diversité structurelle des langues humaines? ", la première chose qu'elle fera, et qu'elle a effectivement entrepris de faire depuis longtemps, est la suivante : elle choisit les types de structure les plus frappants - des styles structuraux-, elle les analyse, les caractérise. Elle explique selon le principe idem per idem ${ }^{25}$ la signification de chaque particularité qu'elle a identifiée, retraduit donc dans le domaine psychologique les phénomènes langagiers, dispose sur cette base les types spirituels des races et des peuples dans des tableaux d'ensemble et teste la justesse de ces tableaux à l'aune de l'ethnologie et de l'histoire. Cette méthode apporterait toute garantie de fiabilité s'il ne lui fallait pas compter avec tant de forces perturbantes qui se dérobent souvent à toute 
observation et, très généralement, à une évaluation précise. Personne ne l'a mise en œuvre avec plus de sagacité et de profondeur que Byrne dans ses Principles of the Structure of Language ${ }^{26}$. Mais il n'a pas accordé, à mon sens, suffisamment d'attention à ces facteurs perturbants, il attribuait dans ses équations une valeur nulle à ce $x$ et il lui arrivait aussi de manier des concepts trop élastiques. Tous ces dangers guettent précisément ce genre de penseur. Dans ma Sprachwissenschaft, j'ai espéré pouvoir les éviter en travaillant à un degré de granularité grossier et en me tenant à ce qui est le plus immédiatement tangible : soit, du côté des langues, à quelques-uns de leurs traits physionomiques les plus saillants et, du côté des peuples et des races, aux masses les plus larges, et à celles qui ont probablement connu le plus longtemps des conditions de vie stables et qui ont en conséquence développé le plus loin certaines dispositions intellectuelles et affectives exclusives. Ce ne fut jamais qu'un essai, mais dont aujourd'hui encore je ne pense pas tant que je l'aurais poussé trop loin, mais plutôt que je ne l'ai pas poussé assez loin. Qu'il restait beaucoup à ajouter dans le détail, je le savais d'emblée ; un seul chapitre eût fourni matière à un gros volume, mais en aucun cas à un travail exhaustif comme Byrne l'envisageait.

Le chemin emprunté était-il réellement le seul praticable ? Et si ce n'est pas le cas, n'y en a-t-il aucun autre qui soit tout aussi sûr, voire plus sûr?

En ce qui concerne leur origine, les forces qui ont façonné les langues sont de deux types, des forces indigènes et des forces étrangères. Sous l'appellation d'indigènes, j'entends toutes celles et seulement celles qui prennent racine au sein de la communauté linguistique elle-même, c'est-à-dire du peuple. Je sais qu'on peut ici distinguer ensuite entre la disposition héritée et cette éducation où c'est le territoire lui-même qui a joué le rôle de maître d'école. Mais les cas où cette distinction est possible sont bien rares.

Toujours est-il que la chose mérite attention, car les migrations sont fréquentes dans l'histoire de l'humanité. Nous aurions alors affaire ici à un facteur perturbant, qui se révèlera, de surcroît, dans la plupart des cas, incontrôlable et d'origine préhistorique. Mais je ne veux pas surestimer son influence. En matière de peuples et de langues, il semble en effet que l'écart entre les dispositions héritées et les nouveaux buts de l'existence, pour autant que ceux-ci soient accessibles à celles-là, se comble assez rapidement. Pour l'un comme pour l'autre, le peuple et la langue, la situation serait préoccupante s'ils ne portaient pas en eux des forces cachées, qui n'attendaient qu'une impulsion pour sortir de leur repos. Mais chez l'un et l'autre, il se peut fort bien aussi que des forces développées s'endorment, faute de n'avoir eu pendant longtemps l'occasion de s'exercer. Nous connaissons des familles de langues de type très homogène et nous en connaissons inversement dont les structures sont d'une étonnante variété, et dans les deux cas, nous pensons en entrevoir la raison. L'unicité ou la diversité des structures linguistiques va de pair, sur le plan ethnologique et historique, avec l'équivalence ou la différence de conditions de vie. Les langues anciennes ne sont pas toujours ici les meilleurs témoins. Et cette science qui creuse le sol à la recherche des langues originaires n'exhume pas précisément le matériel le plus utilisable. Elle peut bien tenter de reconstituer une image du peuple primitif à partir de la langue primitive, sauf qu'elle tournera tout simplement en rond, en prétendant ensuite expliquer la langue primitive à partir du peuple primitif. Elle a en revanche raison d'évaluer les dispositions des races à l'aune de la langue primitive-des dispositions dont le développement a pris des directions différentes chez les peuples 
particuliers, tout en subissant pourtant la pression et l'attraction de forces fondamentalement identiques. Cependant même cette démarche est le cas échéant sujette à limitation, car les caractéristiques partagées par la famille ne sont peut-être significatives que là où elles se sont maintenues vivantes. Le latin a perdu la gradation vocalique en dehors de quelques restes infimes et ses filles ont perdu la flexion casuelle; le persan moderne et, sous des formes étonnamment variées, les langues slaves ont transformé l'ancien système accentuel; dans l'aire indochinoise se sont développés poly- et monosyllabisme, isolation, agglutination et flexion, ainsi que les formes les plus variées de structure phrastique. Nous savons par conséquent qu'en la matière les types langagiers et raciaux ont fait preuve de leur flexibilité. Nous souhaitons dans ce cas nous enquérir de l'origine de cette flexibilité. Et lorsque l'histoire reste sans réponse, lorsqu'elle ne permet pas de deviner ce qu'il faut attribuer à la nouvelle patrie et ce qu'il faut attribuer à l'influence perturbante ou stimulante des peuples voisins, du moins avons-nous obtenu un résultat négatif.

Depuis que, grâce notamment à Hugo Schuchardt et Lucien Adam, notre science étend son indulgence à ces langues hybrides nouvellement nées, il nous est également loisible d'utiliser les mélanges de langues pour nos objectifs. Ce n'est nullement le cas à vrai dire de ces produits informes du commerce international, dont, si je puis dire, il n'est aucun peuple qui intellectuellement les ait investis ou y ait prospéré. Il me faut en conséquence exclure de l'induction des groupes entiers de langues qui me paraissent suspects à cet égard - le groupe des langues mélanésiennes, celui de la Côte-de-l'or en Afrique et d'autres encore. Mais nous savons par ailleurs qu'ainsi ont commencé certaines langues qui comptent aujourd'hui parmi les meilleures. Le bâtard a en l'occurrence été légitimé per subsequens matrimonium, par mariage subséquent, et au sein du nouveau peuple et avec lui, une nouvelle langue s'est développée. Et nous avons alors de nouveau affaire à ce qui nous importe: des structures libres qui se sont développées sur un territoire.

Le matériel de nos inductions se révèle par conséquent plus riche et fiable qu'il n'y paraissait au premier abord. Les langues des peuples civilisés, dont la force s'est déployée dans toutes les directions, sont en revanche des objets d'investigation moins commodes que celles des races inférieures, qui ont développé des dispositions spécialisées. En voilà assez pour la première orientation.

Si je fais abstraction de ces influences perturbatrices étrangères, aussi longtemps qu'elles restent encore étrangères et perturbatrices, ainsi que des langues chez lesquelles il me faut soupçonner de telles influences, je puis dire des autres langues que ce sont des structures organiques libres et que, parce qu'elles le sont, et pour autant qu'elles le soient, toutes leurs parties sont l'une à l'autre en rapport nécessaire. Ceci est évident a priori, il ne saurait en être autrement et c'est pourtant là une thèse très forte. C'est de la même disposition intellectuelle, des mêmes conditions historiques que naît tout ce qu'une langue est et possède : sa phonologie tout comme la formation spécifique de ses mots et de ses outils grammaticaux, tout comme sa syntaxe et le style national, la grammaire comme le lexique.

On peut poser cela comme une thèse et être assuré de l'assentiment général. Il est de même évident que la physionomie des langues présente certains traits particulièrement caractéristiques, notamment des traits lexicaux, stylistiques et syntaxiques. Mais si on va plus loin, si on prétend imiter la tsigane qui interprète l'homme tout entier à partir des lignes de la main, ou un Cuvier qui construisait mentalement tout l'animal à partir 
d'un seul os - si on confronte la théorie aux faits -, alors il apparaît bientôt que nous n'ayons que le triste choix de nous déclarer tout de suite insolvables, ou de bricoler en fonctionnant à crédit jusqu'à ce que la banqueroute se produise d'elle-même.

On ne peut cependant renoncer à une idée nécessaire, offrant une garantie de justesse, au motif que la première tentative de la réaliser a échoué. Il s'agit de la couler dans une forme contrôlable et il n'en est aucune qui soit mieux contrôlable que la forme statistique. C'est à cet endroit que je souhaiterais voir débuter le travail. J'évoquais à l'instant les cas où des langues d'une seule et même famille ont pris des caractères très différents, ce sont maintenant ces autres cas qui sont pour moi intéressants, ceux dans lesquels des langues de souches différentes présentent des traits affines ${ }^{27}$. Dans ma Sprachwissenschaft, j'ai ainsi pu comparer les langues malaises aux langues sémitiques sur le plan syntaxique-et j'aurais pu également mentionner une certaine correspondance dans la formation des mots et des outils grammaticaux. Le fait que des peuples voisins de souches différentes présentent souvent quelque chose de commun dans leur phonologie a plus souvent été observé, y compris par moi-même, et cela paraît explicable. Le fait en revanche qu'en Chine et dans la péninsule du Sud-Est asiatique, trois familles de langues normalement polysyllabiques et agglutinantes, l'indochinoise, la mounda et la malaise, aient des parents monosyllabiques et isolants paraît complètement mystique ${ }^{28}$. Ajoutons-y qu'une différence d'esprit radicale sépare des langues indochinoises le chinois, le birman, ainsi que le siamois et sa famille, lequel ressemble quant à lui tellement à l'annamite, qui est de souche différente, qu'on dirait qu'un même plan de construction a été réalisé, ici en moellons, là en blocs de sable.

Mais il y a une autre observation à peine moins surprenante, en l'occurrence quand deux traits physionomiques, qui n'ont apparemment strictement rien à voir entre eux, se retrouvent appariés aux points les plus divers du monde des langues. Voici l'exemple de ce genre le plus frappant que je connaisse. Le basque en Europe, le tibétain en Asie, le groenlandais et les langues de sa famille en Amérique, ainsi que les langues des aborigènes noirs d'Australie sont passablement éloignés l'un de l'autre dans leur structure grammaticale. Et pourtant les trois premières langues partagent deux traits par ailleurs rares: premièrement, au lieu d'un cas sujet et d'un cas objet, elles possèdent un actif instrumental et un neutro-passif, de sorte que (traduit dans nos concepts) l'objet d'un verbe actif et le sujet d'un verbe neutre, d'une part, le sujet et l'instrument d'un verbe actif, d'autre part, apparaissent chacun au même cas. Deuxièmement, ces langues établissent une nette distinction entre les deux sortes d'attribut adnominal ${ }^{29}$ en antéposant le génitif et en postposant l'adjectif. En termes statistiques : A est corrélé à B dans trois cas sur quatre, B est corrélé à A peut-être dans les trois cinquièmes ou la moitié des cas. La conjonction n'est pas nécessaire, mais elle est néanmoins trop fréquente pour qu'on se contente de l'attribuer au hasard. On a le sentiment d'être sur la trace de deux nerfs sympathiques, qui ne fonctionnent pas systématiquement ensemble et on aimerait donc connaître où et comment ils se sont liés et pourquoi cette liaison est parfois perturbée.

Nous devons probablement nous attendre partout à de telles perturbations occasionnelles, mais aussi à un ensemble de formules énonçant que le phénomène $\mathrm{A}$ est corrélé avec telle probabilité à $\mathrm{B}, \mathrm{C}, \mathrm{D}$, etc., qu'il l'est rarement à $\mathrm{E}$ et ne l'est jamais à $\mathrm{F}$. Et c'est là la statistique que je réclame dans l'immédiat. La question est: est-elle accessible dès maintenant et à quoi nous permettrait-elle d'accéder? 
58 Je puis assurer sans crainte qu'il serait possible de le mettre en place avec nos moyens actuels. Il ne devrait pas être très difficile d'en esquisser le programme. Et les ressources littéraires sont dans une large mesure disponibles, nous n'avons que l'embarras du choix. L'étude des langues conquiert une province après l'autre, elle signale son pouvoir par des manuels toujours plus efficaces. Et néanmoins, l'édifice devrait être érigé sur une base plus large. Chaque type plus important devrait être mis en valeur et pour chaque langue un représentant dûment légitimé devrait servir de répondant. Ce n'est pas en recourant à des extraits schématiques et mécaniques de grammaires tout venant, et parfois vraiment mal venues, qu'on progressera ici. Mais il faudrait justement qu'autant que possible, un grand nombre des langues les plus diverses aient voix au chapitre, davantage que ce qu'un seul individu est réellement capable de maîtriser. Ce travail demande une commission et cette commission demande un programme travaillé jusque dans ses moindres détails, et ce programme demande davantage d'abnégation qu'on ne peut en attendre de la plupart des savants. Ces difficultés devront néanmoins être surmontées.

59 Mais je songe dans ce programme à une sorte de questionnaire couvrant exhaustivement, par catégories, toutes les possibilités grammaticales, de sorte qu'il soit répondu par oui ou non à chaque question. Une telle enquête est difficile pour l'enquêteur lui-même, et parfois aussi pour l'enquêté, mais l'impossible ne sera exigé d'aucun des deux.

Le deuxième acte est purement mécanique, c'est la statistique des conjonctions [Konjunkturen], dont je viens de fournir une rapide illustration. Grâce à cette dernière, nous parviendrions à connaitre avec une exactitude incontestable les traits typiques réellement déterminants. Ce que nous pouvions pressentir depuis longtemps, ce que j'avais moi-même tenté de montrer dans mon livre sur des exemples isolés: ces tendances prédominantes qui s'expriment dans les aspects les plus divers de la vie du langage, ces tendances verraient ainsi leur contenu et leur valeur littéralement mesurés - sous forme quantifiée, comme s'il s'agissait de masses ou de poids. On aurait alors établi les bases d'un calcul de probabilités de très grand intérêt. À partir d'une douzaine de propriétés connues d'une langue, on pourrait en inférer avec certitude une centaine d'autres. Les traits typiques, les tendances dominantes apparaîtraient clairement aux yeux.

61 En clair mais en bref, aussi longtemps qu'il nous est permis de ne parler que de coïncidence et non de liaison systémique. Notre troisième tâche, la plus importante, consiste à établir cette dernière. Et ici la linguistique s'appuiera de son côté sur l'ethnologie et l'histoire, elle y puisera et elle y versera - le percement d'un tunnel entrepris de chaque côté de la montagne. D'un côté, on explique : voici la spécificité de la langue et par conséquent la spécificité de l'esprit national. De l'autre, on conclut: voici les conditions de vie permanentes, voici les expériences historiques antérieures, voici les habitudes et les productions culturelles du peuple, en conséquence, son type intellectuel doit être tel et tel. On entend d'ici les coups de pics de là-bas ; à moins qu'on ait, littéralement, mal creusé la question. Peut-être verra-t-on de nouveau intervenir cette subjectivité du penseur qu'on reproche si volontiers à la linguistique générale et à ses représentants. Mais comme la voilà repoussée bien loin à l'arrière-plan, tandis que s'étend largement la perspective la plus objective qu'on puisse réclamer, celle établie par des données chiffrées! Que l'ouvrage parvienne ne serait-ce que jusque-là, ne serait-ce qu'à une statistique incontestable et la linguistique générale n'aurait plus à 
envier à la linguistique historique la solidité de ses fondements. Et que le travail vînt à se poursuivre, alors le vingtième siècle commençant pourrait voir réaliser ce que les cogitations du dix-neuvième à ses débuts avaient vainement tenté de mettre au monde : une véritable grammaire générale, entièrement philosophique, et néanmoins entièrement inductive.

Berlin, Georg von der Gabelentz*

* Cet article était composé et devait être envoyé à l'auteur pour correction quand survint le choc de l'annonce de sa disparition. Hans Georg Conon von der Gabelentz, académicien et professeur à l'université de Berlin, est mort le 10 décembre 1893 [note du comité de rédaction d'Indogermanische Forschungen].

\section{BIBLIOGRAPHIE}

Byrne, James. 1885. General Principles of the Structure of Language. Londres : Trübner \& Co.

Coseriu, Eugenio. 1967. Georg von der Gabelentz et la linguistique synchronique. Word 23 : 74-100.

Cuvier, Georges. 1812. Recherches sur les ossemens fossiles de quadrupèdes où l'on rétablit les caractères de plusieurs espèces d'animaux que les révolutions du globe paroissent avoir détruites. Tome premier, contenant le Discours Préliminaire et la géographie minéralogique des environs de Paris. Paris : Deterville.

François, Jacques. 2017. Le siècle d'or de la linguistique en Allemagne. De Humboldt à Meyer-Lübke. Limoges : Lambert-Lucas.

Gabelentz, Georg von der. 1881a. Die ostasiatischen Studien und die Sprachwissenschaft. Leipziger Antrittsrede. Unsere Zeit. Deutsche Revue der Gegenwart I : 279-291.

Gabelentz, Georg von der. 1881b. Chinesische Grammatik mit Ausschluss des niederen Stiles und der Umgangssprache. Leizpig : T.O. Weigel.

Gabelentz, Georg von der. 1891. Die Sprachwissenschaft. Ihre Aufgaben, Methoden und bisherigen Ergebnisse. Leizpig : T.O. Weigel.

Gabelentz, Georg von der. 1894. Hypologie der Sprachen, eine neue Aufgabe der Linguistik. Indogermanische Forschungen IV : 1-7.

Gabelentz, Georg von der. 1901 [1891]. Die Sprachwissenschaft. Ihre Aufgaben, Methoden und bisherigen Ergebnisse. $2^{\mathrm{e}}$ éd. Leipzig : Chr. H. Tauchnitz.

Gabelentz, Georg von der. 2016 [1891]. Die Sprachwissenschaft. Ihre Aufgaben, Methoden und bisherigen Ergebnisse. Berlin : Language Science Press. Nlle éd. établie par Manfred Ringmacher \& James McElvenny.

Gabelentz, Hans Conon von. 1832. Élémens de la grammaire mandchoue. Altenbourg : Comptoir de la littérature.

Herder, Rafael \& Benjamin Herder. 1857. Herders Conversations-Lexikon. vol. 5. Fribourg-enBrisgau : Verlag Herder. 
La Grasserie, Raoul de. 1889-1890. De la classification des langues. I : Classification des langues apparentées. II : Classification des langues non-apparentées. Internationale Zeitschrift für Allgemeine Sprachwissenschaft $4: 374-387 ; 5: 296-338$.

Leskien, August. 1875. Hans Conon von der Gabelentz also osterländischer Geschichtsforscher. Wissenschafliche Beilage der Leipziger Zeitung 18 : 105-108.

McElvenny, James, dir. 2019. Gabelentz and the Science of Language. Amsterdam : Amsterdam University Press.

Meillet, Antoine. 1903. Introduction à l'étude comparative des langues indo-européennes. Paris : Hachette.

Owen, Richard. 1848. On the Archetype and Homologies of the Vertebrate Skeleton. Londres : Taylor.

Plank, Frans. 1991. Hypology, Typology : The Gabelentz Puzzle. Folia Linguistica XXV/3-4 : 421-458.

Samain, Didier. 2008. Langues et métalangages : verbe et prédication chez Heymann Steinthal. Cahiers de l'ISL 25 (La structure de la proposition : histoire d'un métalangage ) : 147-166.

Schuchardt, Hugo. 1976 [1928]. Hugo Schuchardt-Brevier. Ein Vademecum der allgemeinen Sprachwissenschaft, Zsgest. u. eing. v. Leo Spitzer. Darmstadt : Wissenschaftliche Buchgeselleschaft.

Streitberg, August. 1892. Von der Gabelentz. Die Sprachwissenschaft. Indogermanische Forschungen 2.1 ( Anzeiger für indogermanische Sprach- und Altertumkunde ) : 1-6.

Walravens, Harmut. 2008. Les recherches sur l'Extrême-Orient au début du XIX siècle, ou Paris, Mecque des orientalistes allemands. Revue germanique internationale $7: 33-48$. [http:// rgi.revues.org/392, consulté le 2/10/2020].

\section{NOTES}

1. Coseriu 1967. Une version allemande de ce texte a été ajoutée aux premières rééditions modernes de Sprachwissenschaft à partir de 1972.

2. Gabelentz 1881b. Peut-être la première grammaire chinoise totalement affranchie du modèle latin.

3. Die Sprachwissenschaft, ihre Aufgaben, Methoden und bisherigen Ergebnisse (Gabelentz 1891, 1901 et 2016).

4. Il n'est pas possible, dans cette brève présentation, de parcourir, fût-ce très sommairement, la littérature secondaire consacrée à Gabelentz en Allemagne et hors d'Allemagne. Parmi les publications les plus récentes, cf. McElvenny 2019, auquel un compte rendu est consacré dans le présent numéro d'HEL.

5. Elle a été achevée par Albrecht von den Schulenburg, qui indique y avoir apporté sa propre contribution mais ne signale pas les passages concernés. En dehors des références postérieures à la mort de Gabelentz, le lecteur en est donc réduit à des indices: le style très personnel de l'auteur et la présence de notions similaires dans d'autres publications. Qu'elles soient dues à l'un ou l'autre auteur, l'étendue des modifications apportées apparaît clairement dans la récente réédition de l'ouvrage (éd. Ringmacher \& McElvenny, 2016), qui les signale par la couleur des polices.

6. La thèse de Coseriu a été violemment attaquée quelques années plus tard par K. Koerner, auquel François (2017) apporte une réponse bien argumentée. François fournit en outre une version bilingue du deuxième texte présenté ici, et d'un passage très semblable qu'on trouve à la fin de Sprachwissenschaft (1901). La différence de style entre les deux textes est en revanche 
perceptible et laisse supposer une intervention de Schulenburg. S'agissant des présentes traductions, depuis longtemps achevées, un concours de circonstances en a retardé la publication après celle du livre de Jacques François. Je le remercie chaleureusement de ne pas s'être formalisé de ce doublon involontaire. J'ai du moins fait en sorte que ma contribution vienne en simple complément du travail, bien plus détaillé, qu'il a consacré à Gabelentz.

7. De la part de l'un des pères du mouvement néogrammairien, le compliment n'est pas à prendre à la légère. On se souviendra au passage que le mandchou servait traditionnellement de langue d'accès aux textes chinois classiques et que la référence à cette langue dans la conférence de 1879 doit donc être comprise comme un hommage implicite à Hans Conon. Il n'est par ailleurs pas anodin que ce dernier ait publié sa grammaire en français, qui était la langue des sinologues du début du siècle. Par cette ligne, les Gabelentz étaient aussi reliés à Paris (voir ci-dessous, n. 9).

8. Cf. Samain $2008: 147-166$.

9. Dans sa conférence, Gabelentz évoque le poste de Wilhelm Schott (1802-1889) à Berlin, mais si Schott fut effectivement le passeur de la sinologie en Allemagne entre la première génération, celle des Allemands de Paris regroupés autour de Rémusat, et celle de la fin du siècle, il vécut plutôt misérablement sans jamais devenir professeur. Une chaire de langues asiatiques avait en revanche été créée à Bonn dès 1816 pour le sinologue Julius Klaproth (1785-1835), mais celui-ci ne l'occupa jamais, préférant rester à Paris. Tout au long du siècle, il y eut en Allemagne des sinologues de valeur, sans oublier Steinthal, mais on peut considérer que la chaire de Leipzig sanctionne l'institutionnalisation, assez tardive donc, de la discipline en Allemagne. Pour un portrait des Allemands de Paris, cf. Walravens 2008.

10. Création d'une chaire de langues est-asiatiques et linguistique générale.

11. Faute de véritable modèle, elle fournissait du moins une représentation intuitive de ce que peut être un système, au point que son usage, explicite ou non et accompagné ou non du cliché "tout se tient ", s'est perpétué au-delà du xix siècle. La question reste ouverte de savoir si le «structuralisme » de la période suivante hérite ou non de cet organicisme-là.

12. Cf. Plank 1991 pour une utile contextualisation. Sans valoir preuve absolue, les thèses développées dans cette conférence et dans l'article suivant suggèrent que, sinon l'expression, du moins le contenu des ajouts introduits dans la réédition de Sprachwissenschaft doit, pour l'essentiel, être attribué à Gabelentz lui-même.

13. Sur Pott et Grasserie, cf. ibid. Même s'il n'y aucune raison de penser que Gabelentz ait eu une connaissance fine des débats de son époque dans les sciences de la nature, rappelons que ces disciplines ont infusé les sciences de l'homme bien au-delà de la métaphore organiciste ou de la théorie de l'évolution. Une notion importante est ainsi la distinction entre homologie (soit l'affinité vraie, à fondement génétique) et analogie (soit l'affinité fonctionnelle - celle qui associe aux poissons les manchots et les mammifères marins par exemple). En d'autres termes, la distinction entre deux types d'apparentement était disponible dans le savoir commun quand les linguistes l'ont développée.

14. « Hypologie der Sprachen, eine neue Aufgabe der Linguistik». Rien ne justifiant dans l'article l'appel à cette notion médicale, il faut lire «Typologie der Sprachen ».

15. Zusammenhang peut se traduire par corrélation. Toutefois la «typologie " de Gabelentz se rapproche davantage d'universaux implicatifs à la Greenberg, puisqu'elle postule l'existence de traits saillants dont découlent les autres. Dans le cas d'une corrélation stricto sensu, l'implication est réciproque (mais nous allons voir que le modèle vient probablement des sciences de la nature).

16. Cf. ici même le compte rendu de McElvenny (2019).

17. Meillet $1903: 407$.

18. Cf. Cuvier : "Tout être organisé forme un ensemble, un Système unique et clos, dont toutes les parties se correspondent mutuellement, et concourent à la même action définitive par une réaction réciproque. Aucune de ces parties ne peut changer sans que les autres changent aussi ; 
et par conséquent chacune d'elles, prise séparément, indique et donne toutes les autres (Cuvier $1812: 58) »$. On croirait lire du Meillet ou un quelconque structuraliste de la période suivante.

19. Les caractères "supérieurs » ou «dominants » servent à établir les grandes classes. Cette proximité est également soulignée par Plank (1991).

20. Version remaniée de la leçon inaugurale faite par l'auteur dans l'aula de l'université de Leipzig le 28 juin 1879 [note de l'éditeur allemand].

21. Gabelentz, qui disait juste auparavant Orientalistik, parle ici de morgenländischen Studien. Morgenland («Levant») pouvait avoir un sens assez vague et celui que lui donne l'auteur n'apparaît qu'à la lecture de l'article. Nous verrons qu'il lui permet de distinguer un MoyenOrient élargi, jusqu'à l'Inde, et l'altérité réelle de l'Extrême-Orient.

22. Al. linguistik. Le mot était déjà ancien mais ne s'est progressivement généralisé qu'au cours du $\mathrm{XIX}^{\mathrm{e}}$ siècle et il tend alors à désigner la grammaire comparée. Le Herders Conversations-Lexikon (Herder \& Herder 1857 : 294) distingue ainsi la philologie et la partie de l'étude du langage qui étudie « les langues scientifiquement comme telles, c'est-à-dire qui étudie les lois selon lesquelles les différentes langues se sont développées tout autant que leur lexique. Il s'agit de l'étude du langage au sens étroit ou linguistique ». Et d'ajouter immédiatement: "La linguistique est une science jeune et encore très imparfaite. Elle doit son origine à des hommes qui ont introduit la connaissance du sanskrit en Europe." Dans ce discours inaugural très calibré, on peut difficilement penser que l'emploi répété de ce mot, qui pouvait faire figure de néologisme, n'ait pas été délibéré. Quoi qu'il en soit, une allgemeine Sprachwissenschaft, une "linguistique générale ", n'était pas normalement qualifiée de Linguistik. L'auteur évoque aussi plus bas une allgemeine Grammatik, une question restée centrale selon lui, nonobstant la caducité des allgemeine Sprachlehren « à la Becker». Tous ces termes désignent, à la date, des réalités distinctes. Lorsque le contexte ne réduit pas d'office la polysémie du français linguistique, la traduction s'efforce de la limiter, en rendant, dans la mesure du possible, sprachlich par langagier.

23. En français dans le texte.

24. Al. Bildungselemente. Les linguistes désignaient plutôt par là les constituants «formels » d'un lexème (e.g. les suffixes), mais cet emploi n'était pas stabilisé. Dans le langage courant, le mot désigne un « composant », voire une « matière première » (chez Marx par exemple).

25. Pour mémoire, cette expression désigne la faute de raisonnement consistant à introduire le definiendum dans le definiens. Exemple d'école : « la logique est la science de la pensée en rapport avec les lois de la logique ».

26. Le titre exact est General Principles of the Structure of Language, deux forts volumes parus 1885. En soi la typologie de Byrne (1820-1897) n'est pas très différente de celle de Steinthal, et les facteurs qu'il évoque sont voisins de ceux mentionnés par Gabelentz, mais, outre des fondements théoriques particulièrement datés, Byrne postule, de manière largement circulaire - idem per idem- une corrélation causale entre traits intellectuels des races, modes de vie et types linguistiques.

27. Al. wahlverwandte Züge. La référence aux Wahlverwandtschaften, aux affinités électives goethéennes, est transparente. Mais on notera aussi la correspondance avec la distinction entre homologie et analogie, introduite par Richards Owen (1848) en théorie de l'évolution.

28. Sic. Il s'agit peut-être d'une coquille. « Mythique »?

29. Pour mémoire, Attribut en allemand correspond à «épithète » et désigne plus généralement les expansions du SN. 


\section{RÉSUMÉS}

L'article contient la traduction et une brève présentation de deux articles importants de Georg von der Gabelentz. Le premier (1881) est une version revue de la conférence inaugurale présentée lors de sa nomination à la chaire des langues chinoise, japonaise et mandchoue à Leipzig. Il montre comment Gabelentz positionne sa discipline face à la grammaire comparée indoeuropéenne qui servait de référent scientifique pour les linguistes. Le second article, publié en 1894 juste après la mort de l'auteur, introduit en linguistique le terme de typologie. En utilisant des modèles probablement issus des sciences naturelles, l'auteur y esquisse un programme d'approche statistique de la typologie, basé sur des conjonctions de traits, qui annonce les universaux implicatifs de la typologie moderne.

This article includes a translation and a short presentation of two major papers by Georg von der Gabelentz. The first one (1881) is a revised version of the inaugural lecture he presented on the occasion of his appointment to the chair of Chinese, Japanese and Manchu languages, University of Leipzig. The paper shows how Gabelentz delimits his own scientific field in relation to the Indo-European comparative grammar, which was considered as the scientific reference among linguists. The second paper was published in 1894 shortly after the author's death and introduces typology in linguistics. By using models that probably originate from natural science, the author outlines a program for a statistical processing of typology, based on conjunctions of characters, which foreshadows the implicational universals of modern typology.

\section{INDEX}

Mots-clés : Gabelentz (Georg von der), grammaire comparée est-asiatique, organicisme, statistique, typologie, universaux implicatifs

Keywords : East-Asia comparative grammar, Gabelentz (Georg von der), implicational universals, organicism, statistics, typology 